involved in splicing in Drosophila did not rescue the effect of unc-75 mutations. However, EXC-7 also localized to nuclear speckles, and its absence led to a cholinergic dysfunction comparable to that of the UNC-75 mutants, indicating that EXC-7 might also be involved in splicing and regulate synaptic function. If both UNC-75 and EXC-7 are involved in splicing, they seem to have non-redundant functions and probably act on different targets. The identity of these targets, the mechanisms that ensure the specificity of UNC-75 and EXC-7 actions in a subset of neurons, and the precise way in which splicing affects neurotransmission remain unknown and are attractive problems for future research.

Juan Carlos López

\section{(2) References and links} ORIGINAL RESEARCH PAPER Loria, P. M. etal. Two neuronal, nuclear-localized RNA binding proteins involved in synaptic transmission. Curr. Biol. 13, 1317-1323 (2003)

FURTHER READING Dredge, B. K. et al. The splice of life: alternative splicing and neurological disease. Nature Rev. Neurosci. 2, 43-50 (2001)

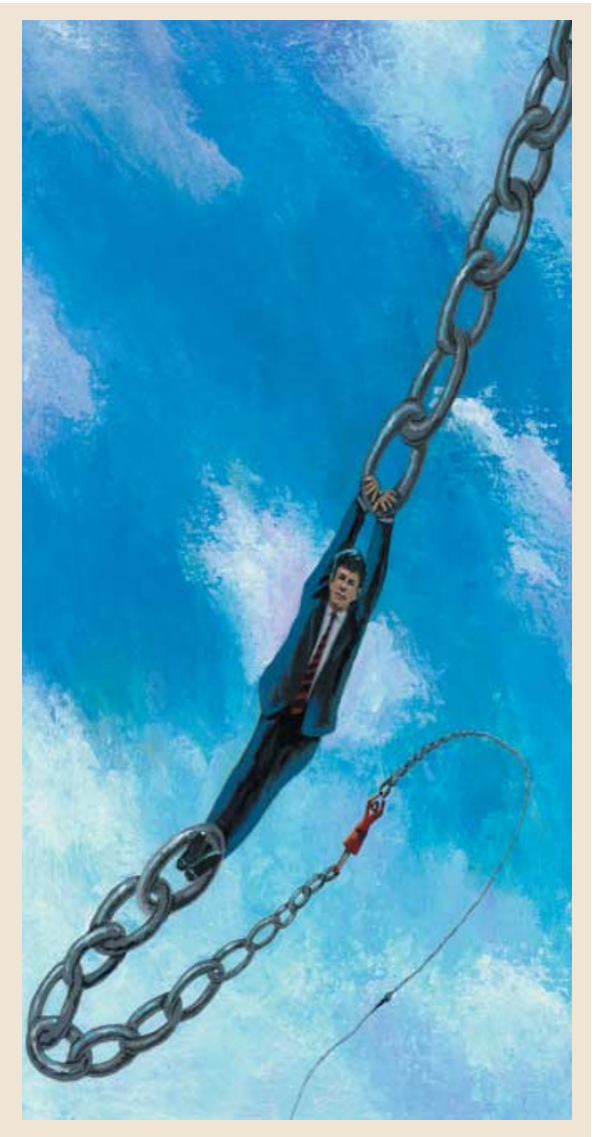

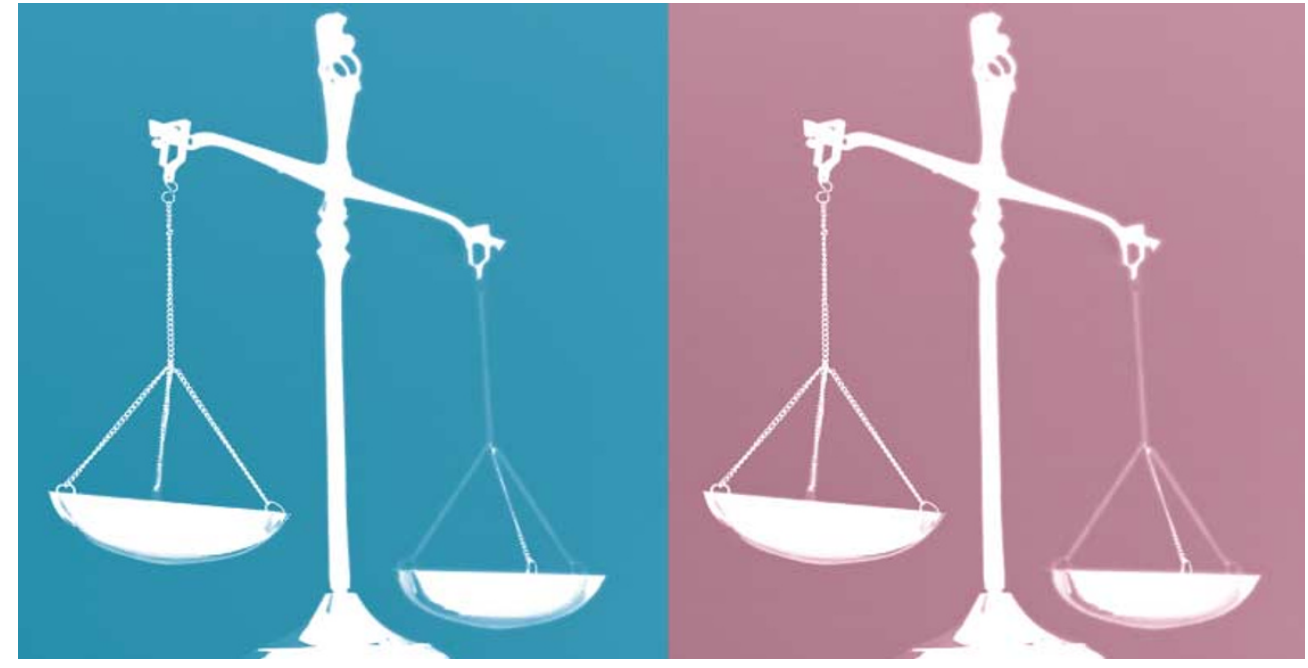

PSYCHIATRIC DISORDERS

\title{
Weighing up the risks
}

The causes of bipolar disorder are far from clear, although family, twin and linkage studies have shown that genetic factors play an important part in its development. Reporting in Nature Genetics, Kakiuchi and co-workers now identify XBP1 - a gene that is central to the endoplasmic reticulum (ER) stress response - as contributing to the genetic risk factor for this condition.

The rate of concordance for bipolar disorder in monozygotic twins is considerably higher than that in dizygotic twins, but some monozygotic twins are discordant - that is, only one of the pair of twins is affected. The biological basis of discordance between monozygotic twins is not fully understood, although several mechanisms have been documented.

Kakiuchi et al. carried out a DNA microarray analysis of lymphoblastoid cells - which have altered signal-transduction systems in patients with bipolar disorder - from two pairs of monozygotic twins who were discordant for the condition. They found that XBP1 and HSPA5 were downregulated in both of the affected twins. HSPA 5 gene expression is known to be induced by the mood stabilizer valproate, and is regulated by XBP1. The team went on to show that a singlenucleotide polymorphism $(-116 \mathrm{C} \rightarrow \mathrm{G})$ in the promoter region of $X B P 1$ that affects a putative binding site for XBP1 itself was more common in Japanese patients with bipolar disorder than in healthy subjects, and was overtransmitted from parents to affected offspring in a collection of samples from people of mixed ethnicity, chiefly of European origin.

Because an XBP1-binding motif seems to be abolished in the risk allele, the group reasoned that the $-116 \mathrm{C} \rightarrow \mathrm{G}$ polymorphism might alter the positive feedback activity of XBP1. They found that XBP1-dependent transcription of the $-116 \mathrm{G}$ allele was significantly lower than that of the $-116 \mathrm{C}$ allele; in addition, in cells with the $-116 \mathrm{G}$ allele, the induction of XBP1 expression in response to ER stress was reduced.

So, the $-116 \mathrm{C} \rightarrow \mathrm{G}$ polymorphism in $X B P 1$ seems to cause a defect in its positive feedback system and to increase the risk of developing bipolar disorder. But do valproate and other mood stabilizers affect this feedback loop? Kakiuchi et al. found that valproate, but not lithium or carbamazepine, could rescue the impaired ER stress response by inducing ATF6, the gene upstream of XBP1.

This study implicates the XBP1 loop of the ER stress response in the pathophysiology of bipolar disorder. The fact that only valproate could reverse the impairment of the XBP1 loop raises the possibility of customizing treatments for patients according to the genetic risk for the condition. Moreover, these findings point to ATF6 as a potential target for the development of mood stabilizers. Interestingly, the authors found that $X B P 1$ was expressed at relatively high levels in the human prefrontal cortex. Further studies of this system could provide insights into the molecular mechanisms of mood. Senior Subeditor, Nature

\section{6) References and links}

ORIGINAL RESEARCH PAPER Kakiuchi, C. et al. Impaired feedback regulation of XBP1 as a genetic risk factor for bipolar disorder. Nature Genet. advance online publication 31 August 2003 (doi:10.1038/ng1235)

FURTHER READING Kato, T. Molecular genetics of bipolar disorder. Neurosci. Res. 40, 105-113 (2001) | Ellgaard, L. \& Helenius, A. Quality control in the endoplasmic reticulum. Nature Rev. Mol. Cell Biol. 4, 181-191 (2003) 